\title{
Semantic Prosody Analysis for Commentary of China's National Publicity Film Based on Appraisal Theory
}

\author{
Ling Zhang \\ Wuhan University of Science and Technology City College, China
}

\begin{abstract}
This paper intends to explore the semantic prosody constructed in the commentary text of China's National Publicity film, by using the appraisal framework, and to explain how the prosody stringing throughout the text assists to achieve the film's communicative goal. Its main theoretical framework is based on Appraisal Theory and also combines the analysis of Tenor and Prosody (prosodic structure) to study the interpersonal meaning of the commentary text of China's national publicity film, aiming to probe into whether the prosody constructed in the text can achieve its communicative goal or not, that is, letting the overseas public know and understand a real and positive image of China, by which a model including three steps, that is, 1) analyzing the Tenor; 2) Analyzing and tagging the appraisal resources; 3) Constructing prosody of the text, is been applied. In data analysis, the author tags all appraisal resources of the text and then probes into their attributions to figure out whether they are consistent with text's communicative goal. The research result proves that as a publicity film, it is reasonable to have the positive resources of Appreciation and Judgment foregrounded in the text and hence to demonstrate a positive image to the addressees.
\end{abstract}

Index Terms - prosody, appraisal theory, tenor, interpersonal meaning, commentary

\section{INTRODUCTION}

Having been influenced by the difference of values and cultural background, the western media always implant bias and distortion in their reports on China, which may give rise to the misunderstandings about China among the overseas public. Correspondingly, with the development of economic and political status on international stage, China needs to enhance its international power of discourse and to change its public relations strategy so as to let the overseas public know and understand China correctly. The year of 2011 marks the official beginning of China's overseas public relation strategy, for the publicity film on its national image oriented to the overseas audiences has been broadcasted on American TV. Has it achieved its communicative goal of introducing China's contemporary situation and positive image, thus to dispel overseas audience's misunderstanding about China? Thus, it is worthwhile studying interpersonal meaning of the film's commentary text, concerning the stances it adopts towards both the material it presents and those whom it communicates.

Martin and White set up the Appraisal System, targeting on explaining interpersonal meaning in terms of three subsystems: Attitude, Engagement and Graduation. Interpersonal meaning is associated with prosodic structure, which is constructed by the appraisal resources in the text; and as the text unfolds, those resources resonate with each other to form certain pattern of mood, in manner of a musical prosody, demonstrating the writer/speaker's attitude, slight or strong (Martin \& White, 2008). Besides, Martin points out a useful Genre theory is the one that will allow for both textual prediction and contextual deduction. That is, given a description of the context, it is possible to predict the meanings at risk and the linguistic features likely to be used to encode them (Eggins \& Martin, 1997). And given the Tenor of the text, we might to some extent predict its interpersonal meaning. The appraisal resources splash across the text, trying to construct the interpersonal meaning in the manner of microstructure, by which the relevant prosody is further constructed by those resources in certain pattern; while analyzing the Tenor can to some extant predict the interpersonal meaning of the text in the manner of macrostructure. Therefore, by combining the Appraisal theory and Tenor analysis, it can better analyze the prosody of interpersonal meaning. This paper adopts the top-down approach for analyzing the prosody of interpersonal meaning, which includes three steps: 1) to analyze the Tenor, figuring out the status of the participants and contact between them to construct macro-level interpersonal meaning of the text; 2 ) to tag and analyze the appraisal resources in the text to map unfolding interpersonal motifs; 3 ) to construct prosody of the text based on Step 2 in order to verify whether the interpersonal meaning constructed corresponds with the interactive goals analyzed in Step 1 or not.

\section{ANALysis of Tenor of the SAmple TeXT}

As one variable of Register, Tenor associated with the interpersonal meaning can be reflected by the appraisal resources. Halliday and Hasan explain that "The tenor of discourse refers to who is taking part, to the nature of 
participants, their statuses and roles: what kinds of role relationship obtain among the participants, including permanent and temporary relationships of one kind or another, both the types of speech role that they are taking on in the dialogue and whole cluster of socially significant relationships in which they are involved" (Halliday \& Hasan, 1989, p.12). Poynton (1989) had also provided a model of the Tenor with respect to social context, which is concerned with the constitution of social roles and relationships and the negotiation of these roles and relationships by speakers. This model identified three dimensions to organize the social relationships, that is, power/status, contact and affect. Christie and Martin (1997) exclude the affect oriented variable out of his tenor variables, and believe that power and solidarity (referred as to status and contact) will be sufficient to generalize appraisal patterns across texts at the level of register. Therefore, in this paper, it merely focuses on the power/status and contact between the text-producer and the addressee.

The addressees of the publicity film are mainly the overseas audiences who have had access to know and understand China mainly from the western media for a long time. The unilateral impart of information may result in their misunderstanding and bias about China. Therefore, partially due to these inaccurate reports, and partially due to the difference of the cultural background and value orientation, many westerners are holding a negative impression on China's image. In order to break their stereotyped and misled impression, efforts must be paid to revivify a true and positive image of China. And only truth and openness can correct the prejudice and touch the soul. The truth means turning down bureaucratic tone. Just as Gao Xiaolong, general director of the film, said, the national image is not equal to the government image, but the daily lives of the public. So, the publicity film bases itself on the reality and demonstrates a colorful and real picture of Chinese citizens' lives to the world. At the beginning part of the commentary, it raises two questions using the first person, that is, "Who are the Chinese? What makes us who we are?" to position the key of the commentary on the ordinary Chinese people's tone. Meanwhile, several quotations, in the form of interview, from both Chinese and foreigners are inserted across the film, which gives an impression of that it is a sincere and face-to-face conversation between the addresser and the addressees. To put it in another word, the film is demonstrated for the overseas audiences guided by our ordinary Chinese people rather than the Chinese government. Therefore, the participants engaged in the communication refer to the ordinary Chinese citizens and the overseas audiences, which does not involve the inequality of the social status, but only the different cultural background and value orientation, or the possible implanted misapprehension about the object the participants are negotiating (here obviously refers to China's image). With respect to the solidarity between the participants of this interactive process, the Chinese people want to let the world know more about China on the basis of objectiveness, reality and mutualunderstanding, try to dispel their misapprehension about the twisted truth, and further align value positions with overseas audiences through sharing feelings with them.

Through analyzing the Tenor of the sample commentary, it may find the communicative goal of the text is to demonstrate the overseas audience a vivid, harmonious and real China with a hope of aligning with them on the shared values and feelings. How is the text constructed to achieve this goal? Can the appraisal resources attributed across the text serve for accomplishment of the communicative goal? With these questions in mind, the paper will explore the appraisal resources in detail in the following sections.

\section{Statistical Analysis OF ApPRAisal ResourCeS}

This paper concerns the interaction of attitude and engagement. In the analysis, the authors attend to appraisal values which are sequenced and made to interact so as to generate particular rhetorical outcomes. All attitude and engagement resources in the sample text have been tagged, and the statistic has been shown in tables and figures in the following sections. As to tagging the appraisal resources, the thesis follows Martin's annotation method to tag the unfolding motifs:

- attitude: affect in italics judgment in bold appreciation is underlined +ve for positive attitude -ve for negative attitude

- engagement in boxing

- graduation in bold + underlining

\section{A. Statistics of Attitude}

In Appraisal System, Attitude is "concerned with feelings, including emotional reactions, judgments of behaviors and evaluation of things" (Martin \& White, 2008, p.35). It involves three semantic regions covering what is traditionally referred to as emotion, ethics and aesthetics. Each region corresponds to Affect, Judgment and Appreciation. Affect deals with resources for construing emotional reactions both positively and negatively towards a person, thing, happening or state-of-affairs (Martin, 2000). Judgment encompasses meanings which serve to evaluate human behavior positively and negatively by reference to a set of institutionalized norms. Appreciation is concerned with the resources for construing the evaluations of things, including things we make, performances we give, and also natural phenomena. Values of Appreciation are properties which attach to the phenomenon under evaluation rather than the human subject 
doing the evaluation (White, 2002). The paper tags three subsystems of Attitude respectively and collects the number of occurrence times of each attitudinal resource. The result is presented in the following table.

TABLE 1:

STATISTIC OF ATTITUDE RESOURCES IN THE TEXT

\begin{tabular}{|l|l|l|}
\hline Attitudinal resources & positive & negative \\
\hline Affect & 14 & 1 \\
\hline Judgment & 37 & 2 \\
\hline Appreciation & 54 & 4 \\
\hline Total & 105 & 8 \\
\hline
\end{tabular}

Table 1 shows that the three attitudinal resources are unevenly distributed. The occurrence of Appreciation resource greatly exceeds the other resources. Of the other two resources, the occurrence frequency of Judgment resources greatly surpasses that of Affect resources. Generally speaking, Appreciation is highlighted by the text producer and thus foregrounded. Obviously, the text tends to impress the audience with good and pleasant image of China. Compared with the positive attitudinal resources, the negative ones rarely appear in the text. This is justifiable since the purpose of a publicity film is to show the positive attitude to the audience.

By further studying the attribution of Affect resources, it was found that they are mainly distributed in introduction and conclusion part. And in introduction part Affect resources are inscribed by the relevant attitudinal expression. For example:

In introduction part:

[1]Our songs of how the earth is our garden and our pride [+ve Affect: inscribed] in surviving our long history suddenly became more vivid and clear.

In conclusion part:

[2]Chinese girl: I wish China will be more beautiful. [+ve Affect: inclination]

The commentary starts with the first-person narration which describes the proud and positive feelings of the Chinese citizens, for they could see their dreams become a reality, and their songs become more vivid. The proud feelings of Chinese people towards their motherland have been inscribed in the abstract narration, which compared with the directly expression is relevantly implicit. By comparison, in the conclusion the text uses direct quotations which directly express the interviewees' inclination affect of wishing their motherland become better and better. The rest of the Affect mainly appears in the quotations from the ordinary Chinese citizens who properly express their satisfaction. The voice from ordinary people makes the Affect more vivid and sincere, and also let the audience construe it as appropriate and reasonable. However, facts should speak louder than affects because facts can justify the affect with proper explanation. That is why Appreciation and Judgment resources are foregrounded in the whole text to evaluate things or behaviors closely related with China or Chinese people. For example:

[3]Foreigner: Every part of China, you have like different culture, I think it is the best place [+ve Appreciation: valuation] in Asia.

The Appreciation resources to some extent are woven into an attitudinal net, by which the text intends to demonstrate the delightful development and pleasant situation of China through the eyes of both Chinese people and foreigners who live in China, and hence to let the overseas audiences know this country once again and endear them to China. The pattern with respect to Judgment resources is analogous with that of Appreciation, dispersing across the text. For example:

[4]30 years opening has been a time of bravely [+ve Judgment: tenacity] facing the new and stepping forward on a path of our own choosing.

Judgment deals with the attitudes to people and the way they behave. Judgment encompasses meanings which serve to evaluate human behavior positively and negatively by reference to a set of institutionalized norms. Under Judgment we may assess behavior as moral or immoral, legal or illegal, socially acceptable or unacceptable and so on. Thus, Martin and White divide Judgment into "social esteem" and "social sanction". "Judgments of esteem have to do with 'normality' (how unusual someone is), 'capacity' (how capable they are) and 'tenacity' (how resolute they are); judgments of sanction have to de with 'veracity' (how truthful someone is) and 'propriety' (how ethical someone is)" (Martin \& White, 2008, p.52). The author finds most of the evaluations in the text express 'social esteem', and if more detailed, on aspect of 'tenacity' and 'capacity'. These evaluations are sometimes directly towards Chinese people's confident and brave character. And it is noteworthy that the text does not shun some controversial issues on China, such as problem of human rights and environment, but positively evaluate the efforts China has devoted to commit its responsibility of being a biggest developing country in the world.

It may indicate that Affect, Judgment and Appreciation resources are carefully chosen, with purpose of renewing audiences' impression on China and hence to form communities of feeling and shared attitudes towards its positive image. However, the question emerging out is how the text manipulates the resources of intersubjective positioning to align themselves with the ideal audience and further to achieve its communicative goal. Then, the author will attend on the Engagement resources of the text and analyzing the stance adopted in the text towards to the value position.

\section{B. Analysis of Engagement}


Engagement deals with sourcing attitudes and the play of voice around opinions in discourse. Martin and White (2008) group system of Engagement into dialogic heterogloss. They suggest that resources of heterogloss can be divided into two broad categories according to whether they 'dialogical expand' (which open up the dialogic space for alternative position) or 'dialogical contract' (which close down the space for dialogic alternatives) in their intersubjective functionality. Under Expand and Contract, four options of dialogistic position- Entertain, Attribution, Disclaim and Proclaim are identified to describe what is at stake when one meaning rather than another is employed (Martin \& White, 2008). This paper takes the two aspects into account, and the attribution of Engagement resources is presented in the following table.

TABLE 2

STATISTIC OF ENGAGEMENT RESOURCES IN THE TEXT

\begin{tabular}{|l|l|l|}
\hline Engagement & Resource & Number \\
\hline \multirow{3}{*}{ Expand } & Entertain & 14 \\
\cline { 2 - 3 } & Attribute & 12 \\
\hline \multirow{4}{*}{ Contract } & Disclaim: counter & 3 \\
\cline { 2 - 3 } & Proclaim: concur & 4 \\
\cline { 2 - 3 } & Proclaim: pronounce & 2 \\
\hline
\end{tabular}

As shown in Table 2, Entertain and Attribute under Expand are prominent in the text, which account for $40 \%$ and $34 \%$ respectively; while, the three sub-resources under Contract take a small part of total number. It reveals that the text mainly adopts the resources of Expand to leave the addresser much space for alternative opinion about the information they are getting.

By using of Entertain, the authorial voice represents the proposition as one of a range of possible positions, which admits alternative opinions. It is noticeable that in the sample text several questions operating as Entertain resources are left for the addressees to judge by themselves on the subjects proposed, hence opening up a space for the possibility of divergent views. For example:

[5] Is tradition a burden, or kind of driving force for development? Do we take our culture heritage too seriously, or not seriously enough? [Engagement: entertain]

Perhaps influenced by some twisted reports or what is so called 'China Threat Theory', many westerners consider China as a foe and its development as a threat. The text producer uses questions as negotiating tone to present the contentious issues to the audiences, which makes allowance for alternative value positions on the propositions, but with the hope of letting the audiences recognize China through their own eyes.

Martin and White (2008) hold that Attribute is most typically achieved through the grammar of directly and indirectly reported speech and thought. On studying Attribute resources in the text, the author found that they are mostly realized by directly reported speech. As discussed before, the resources of Affect, Judgment and Appreciation disperse across the whole text, including the part of frequently-appeared quotations. Therefore, the attitudes demonstrated in this part are attributed to the external voices. Here, in the text, the internal voice is regarded to be from the whole Chinese people. But other voices from some specific Chinese citizens and foreigners, rather than the only and abstract voice (monogloss), can make the opinion and attitude in the film more credible and real. These Attribute resources can be categorized as instances of acknowledge, and interpreted as more or less forthrightly aligning the addressees into the relevant value position. For example:

[6]Chinese Female: "It is the Chinese people that adhere to the environment no matter how hard, no matter how great the set back is we stick to it. Optimism, self-confidence and persistence are the character of Chinese."

Although the resources of dialogic contraction do not attribute as prominently as those of expansion do, they still function as positioning the value stance to some extent. With regard to Counter, it appears three times in the text, and each time cooperates with Entertain. For example:

[7] Some may [Engagement: entertain] argue that under such a huge material and mental stimulation, it would be hard to maintain equilibrium. But [Engagement: counter] after 30 years, the world has seen how much China's profound history and culture essence given leave us the flexibility and unity when facing fast transformation.

By such Entertain + Counter pairings, the text at first opens up the space for alternative positions and validates the addressees' possible contrary viewpoints by admitting that it is understandable and has a rational basis. Then, the addressees are countered with particular beliefs or expectations through counter resources. Thus, such pairings can be seen as gestures towards solidarity in contexts where the writer anticipates disagreement on the part of the addressees, but with the hope of winning them over (Martin, 2000). In a word, these resources are used in the text as strategies to align the addresser with the overseas audiences and let them more likely to accept the attitudes inscribed or invoked by resources of Affect, Judgment and Appreciation.

\section{CONSTRUCtion OF Prosody FOR SAMPLE TEXT}

The Attitude resources operate as the basic elements to demonstrate the feelings of the addresser towards the evaluated; whereas, the resources of Engagement which construct the 'stance' of the appraiser can be seen as the strategy adopted by the addresser to project attitudes on the addressees, and hence setting up a community with the 
shared values. The publicity film uses many positive Appreciation and Judgment resources but relatively less Affects with the request of objectiveness. The resources are mainly deployed within the framework of Entertain and Attribute to construe solidarity with the overseas audience. Combining all appraisal resources together, a semantic prosody is constructed, which can achieve the text's interpersonal function on micro-level.

According to Martin (2004), the structure of interpersonal meaning is realized by three types of prosody: saturation, intensification and domination, and they usually operate together to form a harmonious unity. The realization of saturating prosody is opportunistic, which means relevant interpersonal meaning appears continuously in the discourse; the prosody then manifests where it can. As for intensification prosody, its realization is associated with amplification. The amplification can be realized by repetitions of various kinds to prompt the gradually intensification of relevant interpersonal meaning, which is akin to the use of a pitch movement for highlighting in phonology. Compared with the former ones, the last type of prosody is more complicated. The realization of this kind of prosody is associated with meanings that have other meanings under their scope (Martin, 2004). In the sample text, the foregrounded resources of Appreciation and Judgment are mainly dispersed in the main body, and hence to form the saturating prosody of evaluating positively various aspects of the present China. The structure can be simply presented as follow:

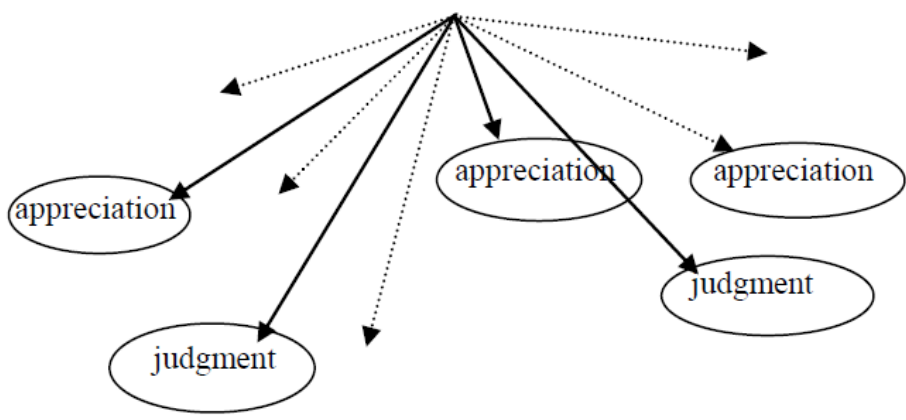

Figure 1 Saturating Prosody of Appreciation and Judgment

With the resources of Affect, they appear mostly in the introduction and conclusion part of the text. Although there is only a small quantity of such resources, they are also attributed in a pattern. It was found that attribution can be construed as forming an intensifying prosody. First of all, the Affect resources attributed in introduction part are inscribed. Then, a few are attributed sporadically in the main body. At last, they are foregrounded in the conclusion part and attributed to several interviewees who directly express their strong wishes for a better China. Therefore, the prosody they construct is like an intensifying one which can be presented as follow:

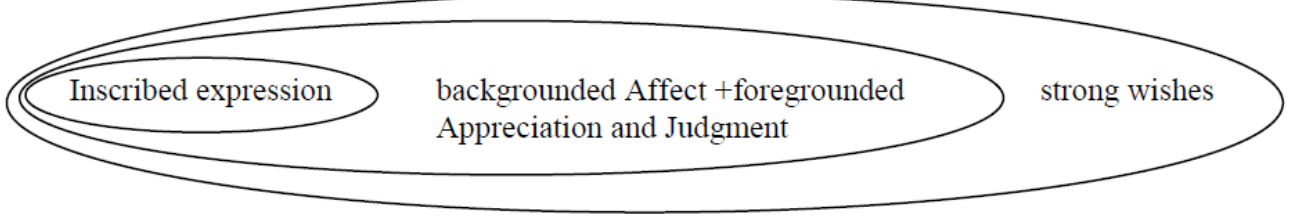

Figure 2 Intensifying Prosody of Affect

Through this prosody, the text-producer intends to evoke the same feelings of the addressees gradually after knowing so many positive aspect of China reflected by foregrounded Appreciation and Judgment resources.

In the sample text, Engagement resources which operate as a strategy to position the stance form a dominating prosody. As mentioned before, the text-producer firstly raised a few questions in the introduction part with the purpose of arousing the audiences' interest and curiosity to know more about China and also inviting the overseas audiences to make their own judgment on those questions being raised after understanding what kind of country China really is through the film. Those questions functioning as Entertain in Engagement system position the text as a negotiable and moderate tone to leave more space for the addressees. Thus, Entertain operates as a dominant strategy to align with the audiences on those positive appreciations and judgments, on which the other resources of Engagement are dependant and serve the same motif. The structure can be presented as follow:

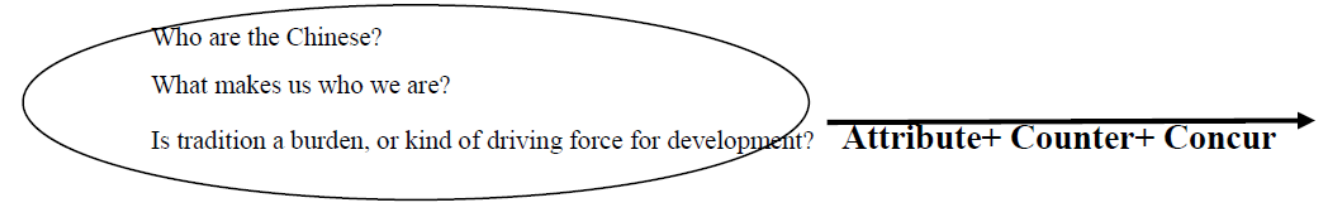

\section{Entertain}

Figure 3 Dominating Prosody of Entertain 
The above three structures are analyzed from the perspective of micro angle of the whole text. But none of them can achieve the interpersonal function of the text without cooperating with the others. They operate as a harmonious unity, running through the text to realize the interpersonal meaning.

\section{SUMMARY}

The communicative goal of the sample text is to align with the overseas audience on the shared value position. The text-producer uses the Entertain and Attribute as main strategy to position the stance of the text and to align with the audiences on the positive image of China reflected by Appreciation and Judgment resources foregrounded in the text. All resources resonate with each other and operate together to form prosodies of interpersonal meaning. These prosodies cooperate with each other to form a harmonious unity to realize the interpersonal function of the text. What should be noted is that whether the goal of a publicity film can be achieved not only depends on the commentary, but also the other factors which should be taken into account, such as the quality of the film itself, the publicity strategy, the respondent of the addressees, the social context and so on so forth. However, the study result shows that from aspect of language used in the commentary, the film proves to be a successful try to show temporary China's positive image to overseas audience and may help to dispel some stereotypes and misunderstandings.

\section{REFERENCES}

[1] Christie, F. \& Martin, J. R. (1997). Genres and Institutions: Social Processes in the Workplace and School. London: Cassell.

[2] Eggins, S. \& Martin, J. R. (1997). Genres and registers of discourse. http://www.grammatics.com/appraisal/ (accessed 24/2/2012)

[3] Halliday, M. A. K. \& Hasan. (1989). Language, Context and Text: Aspects of language in a social-semiotic perspective. New York: Oxford University Press.

[4] Martin, J. R. (2000). Beyond Exchange: Appraisal Systems in English. In Huston, S. \& Thompson, G. (Eds.), Evaluation in Text: Authorial Stance and the Construction of Discourse. Oxford: Oxford University Press, 142-175.

[5] Martin, J. R. (2004). Prosodic 'structure': grammar for negotiation. http://www.wagsoft.com/Systemics/ MartinPapers/index.html (accessed 25/2/2012).

[6] Martin, J. R. \& P. R. R. White. (2008). The Language of Evaluation: Appraisal in English. Beijing: Foreign Language Teaching and Research Press.

[7] Poynton, C. (1989). Language and Gender: Making the Difference. London: Oxford University Press.

[8] White, Peter R. R. (2002). Appraisal: An Overview. http://www.grammatics.com/appraisal/AppraisalOutling/Framed.html (accessed 25/2/2012).

Ling Zhang was born in Lichuan, China in 1986. He received his M.A. degree in Foreign Linguistics and Applied Linguistics from Wuhan University of Science and Technology, China in 2012.

$\mathrm{He}$ is currently a teaching assistant in the Department of Foreign Languages and Law, Wuhan University of Science and Technology City College, Wuhan, China. His research interests include applied linguistics and pedagogy. 